\title{
9 Schlussbetrachtung: Charakteristika von Bewertungsinteraktionen in Pausengesprächen im Theater
}

Die zu Beginn der Arbeit gestellte Frage nach charakteristischen Ausprägungen von Bewertungsinteraktionen in den dieser Arbeit zugrunde liegenden Pausengesprächen im Theater hat die Analyse im empirischen Teil der Arbeit geleitet, deren Ergebnisse sich mit Bezug zu dem in den Daten repräsentierten Theaterpublikum wie folgt zusammenfassen lassen:

Die Analyse von Einstiegen in die Bewertungsinteraktion gibt Aufschluss über den hohen Stellenwert, den die Beteiligten dem Bewerten als kommunikative Aufgabe im vorliegenden Kontext beimessen (vgl. auch Hrncal 2018 sowie Linz/Hrncal/Schlinkmann 2016). Dafür spricht, dass der Einstieg in die Bewertungsinteraktion bei einem Großteil der Gespräche mit dem unmittelbaren Einstieg in das Foyergespräch zusammenfällt oder aber unmittelbar nach dem Sicherstellen der Aufnahme initiiert wird. Ein weiteres Indiz ist die Beobachtung, dass durch einen selbstinitiierten Einstieg in die Bewertungsinteraktion den eigentlich beschreibenden vorangehenden Äußerungen des Gegenübers der Status einer Erstbewertung zugeschrieben wird, ebenso wie die Reaktionen der Gesprächspartner auf eingeforderte Einstiege in die Bewertungsinteraktion, unter anderem mit einer ersten Bewertung als Antwort auf die offene Ein-WortFrage „und?“.

Der mit der Unvorhersagbarkeit der Reaktion des Gegenübers verbundenen Face-Bedrohung gehen die Beteiligten aus dem Weg, indem sie ihr Gegenüber durch Bewertungsunterstellungen oder die Einforderung einer Bewertung zu einer Stellungnahme verpflichten (vgl. Auer/Uhman 1982). Sich selbst räumen sie damit die Möglichkeit ein, erst die Stellungnahme und damit auch die Selbstpositionierung des Gegenübers abzuwarten und in einem dritten Zug ihre eigene Meinung äußern und die mit der Erstbewertung indizierte epistemische Autorität für sich beanspruchen zu können. Ein Bewertungen unmittelbar angeschlossenes Rückversicherungssignal wie ne, gell oder auch oder wird als Verfahren eingesetzt, um die mit der (Erst-)Bewertung beanspruchte epistemische Autorität in gleichem Maße auch dem Gesprächspartner zuzuschreiben. Obwohl die Beteiligten sich durch die Einforderung einer (bewertenden) Stellungnahme ihres Gegenübers vorerst vor einer Erstbewertung und der damit verbundenen Face-Bedrohung schützen, so zeigen die in dieser Arbeit analysierten Bewertungseinstiege doch auch, dass bereits in einer Einforderung zu einer Stellungnahme durch deren prosodische Konturierung Wertung indiziert werden kann 
und so die eingeforderte Erstbewertung zu einer Zweitbewertung wird. Diese Beobachtung widerlegt in Teilen die von Auer und Uhmann (1982) konstatierte Funktion von Bewertungseinforderungen, der mit der Unvorhersagbarkeit der Reaktion des Gegenübers verbundenen Face-Bedrohung aus dem Weg zu gehen: Die Vorhersagbarkeit der Positionierung des Einfordernden wird durch dessen mit Wertung aufgeladene Nachfrage für den Aufgeforderten erleichtert. Wird also die Bewertungseinforderung bereits paraverbal mit Wertung aufgeladen, so könnte der Aufgeforderte seine Zweitbewertung entsprechend der in der Bewertungseinforderung indizierten Wertung zuschneiden, Nichtübereinstimmung vermeiden und möglicherweise eine Face-Bedrohung umgehen. Auf eine Einforderung hin realisierte Erstbewertungen in Form einer (subjektivierten) Deklaration mit Rückversicherungssignal werden vom Gegenüber als Zustimmung mit anschließender neuer Erstbewertung, als neue Erstbewertung ohne Zustimmung oder als Erläuterung der Erstbewertung mit anschließender Globalbewertung des Theaterstücks realisiert.

Bei selbstinitiierten Bewertungen greift die Möglichkeit, das Gegenüber zu einer Erstbewertung zu verpflichten, nicht. Dennoch stehen den Beteiligten andere Möglichkeiten zur Verfügung, um mit der potenziellen FaceBedrohung, die mit Erstbewertungen verbunden ist, umzugehen, etwa die sprachliche Realisierung einer Äußerung, die eine Lesart als Beschreibung oder als Bewertung zulässt, die sprachliche Realisierung in Form eines nicht erfüllten Wunsches oder einer nicht erfüllten Erwartung sowie erlebensdeklarative Äußerungen. Lexikalische Elemente wie Heckenausdrücke dienen der Abschwächung einer Bewertung und der Markierung einer Positionierung als potenziell heikel und Face-bedrohend. Auch trägt die äußerungsinitiale, -interne oder -finale Subjektivierung einer Bewertung zur Abschwächung bei, indem durch finde/ fand ich oder glaube ich die Bewertung als nur für den Sprecher gültig und unsicher gerahmt und so weniger angreifbar gemacht wird. Neben Elementen des Hedgings und Subjektivierungen greifen die Beteiligten auch auf Markierungen des Nichtwissens wie weiß nich oder keine Ahnung zurück. Diese Unschärfemarkierer (vgl. König 2014) können einerseits als Disfluenzmarker im Rahmen von Formulierungsarbeit dienen, andererseits auch indizieren, dass den Sprechern weitere Begründungen ihrer Bewertungen, Deutungen oder Beispiele aktuell nicht zugänglich sind. Durch diese Formen der Realisierung von Bewertungen beziehungsweise die Markierung ihrer Bewertungen als heikel indizieren die Beteiligten fortwährend die Prestigeträchtigkeit und die damit verbundene Angreifbarkeit ihrer Positionierungen im Kommunikationsraum „Kunst“ (vgl. Müller/Kluwe 2012: 5).

Neben der Indizierung sprachlicher Vagheit durch die oben genannten sprachlichen Mittel wird auch anhand der thematischen Verläufe von Bewertungsinteraktionen nachvollziehbar, wie die Beteiligten mit der potenziellen 
Face-Bedrohung und der Gefahr, sich zu blamieren beziehungsweise unangenehm aufzufallen, umgehen (vgl. Filk/Simon 2010, Hausendorf 2005, Schwanitz 1999). Dass bei eingeforderten oder selbstinitiierten Erstbewertungen oftmals Teilaspekte des Theaterstücks (unter anderem das Bühnenbild, einzelne Schauspieler, das Kostüm, die Musik etc.) als Bezugsobjekt in den Fokus der Bewertung genommen werden, die nicht notwendigerweise kunstheoretisches Wissen erfordern, spricht dafür, dass es sich bei diesen Bezugsobjekten um sichere Themen für einen Einstieg in die Bewertungsinteraktion und Kunstkommunikation handelt. Diese These bestärkt auch die Beobachtung, dass auf ein solches Thema zurückgegriffen wird, wenn beispielsweise Deutungsversuche des im Theaterstück Rezipierten auf einer metareflexiven Ebene ins Stocken geraten. Wird dennoch eine globale beziehungsweise pauschale Vorwegbewertung (vgl. Kindt 2007) vorgenommen, so wird das Bezugsobjekt, oftmals durch die Proform es/das vage gehalten, erst im weiteren Verlauf der Bewertungsinteraktion oder gar nicht konkretisiert, und in letzterem Fall stillschweigend vorausgesetzt, dass sich beide Gesprächspartner mit ihren Bewertungen auf dasselbe Objekt beziehen. Auch die Wahl nicht eindeutig negativ oder positiv konnotierter evaluativer Adjektive wie krass oder interessant kann die Beteiligten (zumindest vorerst) davor schützen, sich hinsichtlich des Bewertungsgegenstands eindeutig positionieren zu müssen.

Wie thematische und sequenzielle Verläufe Hand in Hand gehen und wie das Bewerten von den Beteiligten im gemeinsamen Vollzug interaktiv realisiert wird, wurde in Kapitel 8.2 dargestellt. Hinsichtlich der sequenziellen Struktur zeigen sich einige Abweichungen zu den von Pomerantz (1984) sowie Auer und Uhmann (1982) konstatierten Strukturen: In vielen Fällen zieht eine Erstbewertung als zweiten Schritt keine eskalierte, niveaugleiche oder deskalierte Zweitbewertung (mit einer eventuellen Konkretisierung des Bezugsobjekts) nach sich. Zustimmung wird stattdessen über minimale Formen wie ja, das stimmt oder genau kommuniziert (vgl. hier auch Lindström/Heinemann 2009). Bleibt eine Reaktion des Gegenübers gänzlich aus, führt dies entweder zu einer Wiederholung der Erstbewertung durch den gleichen Sprecher oder zu einer erneuten Bewertung. Dabei ist ein Wechsel des Bezugsobjekts möglich, zumeist von der globalen Ebene hin zur ästhetisch-performativen Ebene. Dies spricht nochmals dafür, dass in der Perspektive der Beteiligten Teilaspekten des Theaterstücks der Status eines sicheren Bezugsobjekts zugeschrieben wird und ein Wechsel auf die ästhetisch-performative Ebene eine Strategie darstellt, um mit der potentiell heiklen und Face-bedrohenden Situation umzugehen. Wird eine Zweitbewertung realisiert, so wird dabei möglicherweise ein anderes Bezugsobjekt entweder auf der gleichen oder einer anderen Ebene in den Fokus gerückt. In den analysierten Gesprächen schließen die Beteiligten an 
Bewertungen (Wahrnehmungs-)Beschreibungen, einen Abgleich von Wahrnehmungen, Deutungen, Selbstzuschreibungen oder den Übertrag auf die gesellschaftliche Ebene an. Wird von den Beteiligten ein Übertrag im Stück präsentierter Inhalte auf die gesellschaftliche Ebene außerhalb der Welt des Stücks initiiert, schließt sich an eine Bewertung auf der inahltlichen oder ästhetisch-performativen Ebene des Stücks zumeist erst ein Bezug zu eigenen Erfahrungen aus der persönlichen Alltagswelt an, bevor ein Bezug zur gesellschaftlichen Wirklichkeit vorgenommen wird. Bewertungen dienen den Beteiligten ebenso als Anstoß zur Selbstreflexion (vgl. auch Knape 2016) oder einer szenario-ähnlichen Projektion im Stück aufgeführter Zusammenhänge in die gesellschaftliche Wirklichkeit. Dies gibt einen wichtigen Hinweis auf die Rolle von Bewertungen für die Aneignung der im Theaterstück rezipierten Inhalte: Bewertungen können Deutungen, Selbstreflexionen, Projektionen sowie den Übertrag der präsentierten Inhalte auf die Ebene der gesellschaftlichen Wirklichkeit, also die Welt außerhalb des Theaterstücks, initiieren und die Beteiligten dazu anregen, sich selbst und die gesellschaftlichen Verhältnisse in Frage zu stellen sowie sich im gesellschaftlichen Gefüge zu verorten. Bewertungen tragen somit zur expliziten Aneignung des auf der Bühne Präsentierten bei (vgl. Faber 2001). Zudem bietet das Theaterstück mit seinen Inhalten als potenzielle Bewertungsgegenstände den Beteiligten die Gelegenheit, aufgrund divergierender Bewertungen und Meinungen hinsichtlich ihrer Alltagswirklichkeit herrschenden Dissens auszutragen, ohne dass dies mit der „Gefahr eines Konfliktes mit unabsehbaren Konsequenzen“ (Lüddemann 2007: 36) verbunden ist.

Dass in den Pausengesprächen eine Präferenz für Übereinstimmung vorliegt, wie sie bereits von unter anderem Auer und Uhmann (1982), Pomerantz (1984) sowie Goodwin und Goodwin (1992) für private Alltagsgespräche herausgestellt wurde, wird an der sprachlichen Realisierung von übereinstimmenden und nicht-übereinstimmenden Bewertungen deutlich: Während übereinstimmende Bewertungen direkt, ohne Verzögerungen und Abschwächungen realisiert werden, werden nicht-übereinstimmende Bewertungen von Disfluenz- beziehungsweise Dispräferenzmarkern (vgl. Stukenbrock 2013) wie Verzögerungen, Pausen, Heckenausdrücken usw. begleitet. Allerdings kann die Präferenz für Übereinstimmung auch in eine Präferenz für Nichtübereinstimmung umschwenken. Ein Präferenzwechsel ist dann daran zu erkennen, dass Nichtübereinstimmung direkt und nicht durch Dispräferenzmarker abgeschwächt realisiert wird (vgl. dazu auch Kotthoff 1993 und Lerner 1996).

Nichtübereinstimmung hinsichtlich einer Bewertung tritt in den Pausengesprächen ebenso auf wie Übereinstimmung. Eine Perspektivendivergenz der Beteiligten kann bezüglich der ästhetischen Qualität von Einzelaspekten oder der gesamten Inszenierung des Theaterstücks vorliegen. Dynamik und Intensität 
von Nichtübereinstimmung können zwischen einer abgeschwächten und einer starken Ausprägung ohne jegliche Abschwächung changieren. Nichtübereinstimmung kann sich über ein gesamtes Gespräch (global) erstrecken oder auf kleinere Episoden (lokal) begrenzt sein. Sie wird nicht aufgelöst, wenn beide Gesprächspartner auf ihren divergierenden Bewertungen beharren, ihr Gegenüber nicht zu einer Zustimmung der Bewertung eines Teilaspekts bewegen können oder dies erst gar nicht versuchen und keine weiteren Maßnahmen ergreifen, die zu einer Konzession des Gegenübers führen könnten. Zudem kann Nichtübereinstimmung aufgelöst werden, indem die Erstbewertenden die Zweitbewertenden durch weitere Argumente doch noch von ihrer Bewertung zu überzeugen versuchen, das heißt zu einer Konzession bewegen und die Bewertung doch noch zustimmungsfähig machen. In diesem Zusammenhang rückt auch der Aspekt der Begründung einer Bewertung und damit verbunden auch deren Gültigkeit in den Fokus. Dass Bewertungen nicht notwendigerweise begründet werden müssen, wurde bereits in Kapitel 2.1.2 - allerdings für das Bewerten allgemein - ausgeführt. Wie sieht es nun aber in den vorliegenden Pausengesprächen aus, die in einem semi-institutionellen und semi-öffentlichen Rahmen stattfinden, und in denen es auch darum geht, Wissen zu demonstrieren und sich als gebildet darzustellen?

Vor dem Hintergrund der im empirischen Teil der vorliegenden Arbeit dargestellten Analyseergebnisse sind die bereits in Kapitel 2.1.2 dargelegten folgenden beiden Positionen von Ripfel und Keller kritisch zu betrachten. Nach Ripfel (1987) erscheint die Kompetenz des bewertenden Sprechers, die auf seinen Erfahrungen, Kenntnissen oder Fähigkeiten basieren kann, dafür ausschlaggebend zu sein, ob seinem Urteil Gültigkeit zugeschrieben wird oder nicht. Außerdem spielt für Ripfel (1987) der Faktor eine Rolle, inwiefern Bewertungen rational erklärbar und begründbar sind, das heißt inwiefern die der Bewertung zugrunde liegende Vergleichsbasis offengelegt wird. Die rationale Erklärbarkeit und Begründbarkeit von Bewertungen stellt bei Geschmacksurteilen allerdings ein Problem dar, da die dem Geschmacksurteil zugrunde liegenden Wertvorstellungen nicht notwendigerweise für andere relevant sind (vgl. Ripfel 1987) oder, so Keller (2008), Geschmacksurteile nicht das Ergebnis einer reflektierten Abwägung sind und die dem Geschmacksurteil zugrunde liegenden Wahrheitsbedingungen einzig und allein dem Sprecher zugänglich sind. Diese Standpunkte lassen sich im Zuge der Beantwortung der oben aufgeworfenen Frage den Ergebnissen der in dieser Arbeit durchgeführten Analyse folgendermaßen gegenüberstellen: Erstens wird in den Pausengesprächen die mit den Bewertungen beanspruchte Gültigkeit bereits in der Realisierung der Bewertung durch eine äußerungsinitial, -intern oder -final vorgenommene Subjektivierung indiziert (siehe Kapitel 7.1). Inwiefern die Bewertung für andere gültig ist, wird also be- 
reits von den Bewertenden vorweggenommen (dies gilt auch im Fall der nachträglichen Subjektivierung von Bewertungen in Form einer Prädikation, die vorerst einen Allgemeingültigkeitsanspruch erheben). Zweitens werden Bewertungen in den vorliegenden Gesprächen in großen Teilen begründet, ohne dass die Beteiligten ihre Äußerungen - etwa durch die Konjunktion weil - explizit als Begründung rahmen.

Als Begründungen werden von den Beteiligten

- Belehrungen,

- Erlebensdeklarationen,

- Erfahrungsbeschreibungen und Moralisierungen,

- Beispiele,

- Projektionen,

- Vergleiche und Kontrastierungen sowie

- Geschmacksbekundungen

angeführt. Diese Begründungen können dazu führen, das Gegenüber von einer Bewertung zu überzeugen, ohne dass explizit eine Vergleichsbasis oder Wertmaßstäbe offengelegt werden (siehe Kapitel 7.5). Drittens werden auch Geschmacksurteile (teilweise explizit durch weil) begründet, beispielsweise durch eine reflektierte Abwägung im Rahmen einer Selbstzuschreibung, die den anderen Beteiligten die dem Geschmacksurteil zugrunde liegenden „Wahrheitsbedingungen“ (vgl. Keller 2008) zugänglich und so die Bewertung nachvollziehbar macht (siehe Kapitel 7.5).

Dass es in keinem der Gespräche zu einer ernsthaften Auseinandersetzung aufgrund divergierender Bewertungen kommt - im Gegensatz zu privaten Alltagsgesprächen, in denen es durchaus zu Streit und zur Eskalation kommen kann (vgl. Keppler 1994) - spricht für den bereits in Kapitel 4.2 angesprochenen kooperativen Charakter der Pausengespräche (vgl. auch Habscheid 2018). Dieser kooperative Charakter wird von den Beteiligten etabliert, indem sie

1. sich selbst oder den anderen als relativen Laien beziehungsweise Experten positionieren,

2. Nichtübereinstimmung aushandeln, ohne diese auf die persönliche Ebene $\mathrm{zu}$ übertragen, oder nicht überwindbare divergierende Bewertungen aushalten und damit auch keine Abgrenzung nach innen, das heißt von den anderen Beteiligten innerhalb der Gesprächsgruppe forcieren,

3. ihren Gesprächspartnern (neue) Beteiligungsmöglichkeiten einräumen, wenn diese sich (beispielsweise aufgrund von Nichtwissen) Bewertungsund Deutungsaushandlungen entziehen (siehe Kapitel 8.2 und 8.3),

4. Nichtwissen explizieren und sich (teils ohne dies eingefordert $\mathrm{zu}$ haben) belehren lassen, 
5. Wissensasymmetrien durch Belehrungen oder Erfahrungsschilderungen zu beheben versuchen und

6. so mit der Praxis der Kunstkommunikation weniger Vertraute sukzessive an diese Praxis heranführen (siehe Kapitel 8).

Mit dem Bewerten ist, das wurde bereits deutlich, auch das Demonstrieren und Aushandeln von Wissen beziehungsweise des Wissensstatus der Interagierenden (vgl. Deppermann 2015) verbunden. In Kapitel 4.2 wurde mit Steinig (2016: 71) darauf verwiesen, dass die Demonstration von Wissen einen wichtigen Bestandteil bildungssprachlichen Handelns ausmacht. Sich als gebildet zu positionieren impliziert auch, über Strategien zu verfügen, mit Nichtwissen umzugehen (vgl. Steinig 2016: 71). Wie die Beteiligten in den dieser Arbeit zugrunde gelegten Pausengesprächen mit (aufgedecktem) Nichtwissen im Kontext der Kunstkommunikation umgehen und inwiefern sie Wissen in ihren Bewertungsinteraktionen überhaupt relevant setzen („doing gebildet“, Steinig 2016), konnte in Kapitel 8.2 und 8.3 vor allem anhand der Analyse von Verläufen von Bewertungsinteraktionen und der Aushandlung nichtübereinstimmender Bewertungen nachgezeichnet werden.

Das Pausengespräch als Teil der geselligen Veranstaltung des Theaterbesuchs ermöglicht es, Wissen auszutauschen, voneinander zu lernen und mit dem heiklen, potenziell Face-bedrohenden und prestigeträchtigen Charakter der Praxis der Kunstkommunikation umzugehen. Die örtlichen Gegebenheiten bieten zum einen Infrastrukturen, um sich von der möglicherweise anstrengenden und belastenden Rezeptionserfahrung zu erholen (siehe Kapitel 8.4.1 und Habscheid/Linz 2018), zum anderen liefern sie den Beteiligten Material für scherzhafte Kommunikation, den Wechsel hin zu Small Talk-Themen (im weiteren Sinne), die der Beziehungsgestaltung dienen (siehe Kapitel 4.1 sowie Habscheid 2018) und die Beteiligten von einer fortwährenden Aufrechterhaltung der Kunstkommunikation entbinden können. Auch die in einigen Gesprächen extensiv genutzte Ressource der Reinszenierung rezipierter Inhalte, indem sich die Beteiligten unter anderem in das von ihnen rezipierte Theaterstück projizieren und dort die Rolle einer Figur übernehmen, um diese einer Bewertung zugänglich zu machen oder eine Bewertung zu begründen, dient der humorvollen Unterhaltung aller am Gespräch Beteiligten. Zudem tragen die Reinszenierungen zur psychischen Entlastung der Sprecher bei, die während der Rezeption des Theaterstücks in dieser exaltierten Form nicht möglich ist.

Die institutionell bedingte zeitliche Begrenzung der Theaterpause kann allerdings auch dazu führen, dass der Austausch über das gerade im Theaterstück Rezipierte von den Beteiligten abgebrochen wird und so für die Aneignung des Rezipierten unter anderem relevante Bewertungs- und Deutungsaushandlungen 
nicht zu Ende geführt werden (können). Die Reaktion der Beteiligten auf das Pausenende, das durch ein akustisches Signal, die Ankündigung einer Theatermitarbeiterin oder durch das Fortführen des Theaterstücks im Foyer markiert wird, indiziert ihre Überraschung (siehe Kapitel 8.4.1) und dass das Pausenende für sie nicht exakt vorhersehbar ist (vgl. auch Habscheid/Linz 2018). Die Analyse der Beispiele in Kapitel 8.4.1 hat gezeigt, dass die Markierung des Pausenendes nicht notwendigerweise zu einem Abbruch des aktuell behandelten Themas führen muss, sondern dass dieses - beispielsweise im Falle des Fortführens des Theaterstücks im Foyer - als Ressource für die Initiierung einer neuen Bewertungsinteraktion dienen kann. Selbstinitiierte Ausstiege aus der Bewertungsinteraktion werden in den in Kapitel 8.4.2 angeführten Beispielen durch Disfluenzmarker eingeleitet und mit einem Themenwechsel (unter anderem mit Bezug auf private Aspekte oder die mit der sozialen Veranstaltung zusammenhängenden Umstände der Gespräche, zum Beispiel das Versorgen mit Getränken, die Positionierung im Theatergebäude, andere anwesende Personen) vollzogen. Die beim Ausstieg von den Beteiligten als abgeschlossen gerahmte Bewertungsinteraktion kann im weiteren Gesprächsverlauf wieder aufgegriffen werden.

Bewertungsinteraktionen in der Pause zwischen den Teilen eines Theaterstücks unterscheiden sich von Bewertungsinteraktionen in Gesprächen im Anschluss an die gesamte Rezeption eines Theaterstücks in nur wenigen Punkten: Eine endgültige Bewertung des gesamten Theaterstücks ist erst im Anschluss an die gesamte Rezeption möglich. Das heißt, dass es möglicherweise dazu kommen kann, dass bereits in der Pause realisierte Pauschalbewertungen der erst in Teilen rezipierten Inszenierung im Anschluss an die Gesamtrezeption revidiert werden (vgl. Hrncal/Gerwinski 2015). Des Weiteren wägen die Beteiligten in der Pause im Falle einer (extrem) negativen Gesamtbewertung ab, ob sie sich den zweiten Teil des Stücks überhaupt noch anschauen (siehe Kapitel 8.4.1). Dieser Punkt ist in den Gesprächen im Anschluss an die Gesamtrezeption des Theaterstücks obsolet. In Gesprächen in der Pause des Theaterstücks finden ferner Projektionen der weiteren Inszenierung des Theaterstücks statt oder es werden Wünsche über die Art und Weise des weiteren Verlaufs des Theaterstücks geäußert.

Die Analyse von Bewertungsinteraktionen hat gezeigt, dass das Bewerten für die an den Pausengesprächen Beteiligten eine zentrale kommunikative Aufgabe darstellt und somit nicht nur bei der Aneignung bildender Kunst, sondern auch bei der Aneignung performativer Kunst eine tragende Rolle spielt. Die Aneignung durch Bewertungen geschieht nicht nur stillschweigend (vgl. Schwanitz 1999) während der Rezeption des Theaterstücks, sondern auch und vor allem in der gemeinsamen Anschlusskommunikation 
mit anderen. Diese Anschlusskommunikation zeichnet sich, so wurde deutlich, durch einen höchst kooperativen und vergemeinschaftenden Charakter aus, indem sich die Beteiligten bei der Aneignung unterstützen: Sie beheben Wissensasymmetrien und integrieren ihre Gesprächspartner (wieder) in die Kommunikation über Kunst, wenn diese Schwierigkeiten zeigen, mitzuhalten (vgl. Steinig 2016). Auch lassen sie sich an ihrem inneren Erleben während der Rezeption des Theaterstücks teilhaben. Pausengespräche stellen somit nicht nur für die Aneignung des Theaterstücks, sondern auch für die Konstitution des Theaters einen wichtigen Teil dar: Indem die Beteiligten die Pausengespräche nutzen, um das im Theaterstück Präsentierte mit ihren eigenen Erfahrungen und der gesellschaftlichen Realität in Verbindung zu bringen, sich selbst und ihre Rolle in der Gesellschaft zu reflektieren und in Frage zu stellen, Lernprozesse zu ermöglichen und an die Praxis der Kunstkommunikation heranzuführen, setzen sie selbst die Bedeutung beziehungsweise den Nutzen des Theaters für ihre eigene Alltagswirklichkeit relevant.

Mit den vorangehend dargestellten Ergebnissen knüpft die vorliegende Arbeit an Forschungsarbeiten zu konversationsanalytischen Studien von alltäglichen privaten und institutionellen Bewertungsinteraktionen an und erweitert die in ihnen gewonnenen Erkenntnisse um einen weiteren, bisher in der Konversationsanalyse noch nicht erforschten institutionellen Bereich. So konnten einige der von Pomerantz (1984) sowie Auer und Uhmann (1982) konstatierten strukturellen Ausprägungen von Bewertungsinteraktionen, wie eine Präferenz für Übereinstimmung oder eine konditionelle Relevantsetzung einer Zweitbewertung durch eine Erstbewertung bestätigt werden, andere hingegen in Teilen widerlegt oder erweitert werden: Die Präferenz für Übereinstimmung ist in den dieser Arbeit zugrunde liegenden Gesprächen kein grundlegendes konversationelles Prinzip, Bewertungseinforderungen können aufgrund ihrer prosodischen Konturierung bereits Erstbewertungen indizieren und Erstbewertungen müssen nicht notwendigerweise mit einer Zweitbewertung quittiert werden (siehe dazu auch Kapitel 2.3.2).

Ferner erweitert die vorliegende Arbeit den Erkenntnisstand zum Bewerten als eine zentrale Aufgabe in der Praxis der Kunstkommunikation. Während sich bei parallel zur Betrachtung eines Kunstwerks stattfindenden Bewertungsinteraktionen das Bezugsobjekt im gemeinsamen Wahrnehmungsraum der Interagierenden befindet und die Betrachter fortwährend (unter anderem auch durch Zeigegesten) darauf verweisen können, sind in der Anschlusskommunikation im Theaterpausengespräch Verweise auf das Bezugsobjekt nur retrospektiv möglich. Dies führt dazu, dass das Bezugsobjekt der Bewertung in vielen Fällen zuerst rekonstruiert werden muss (vgl. Schlinkmann 2018), um es einer Bewertung zugänglich machen zu können. Auch für den Forschungsstand der zumindest nur semi-öffentlichen, nicht-professionellen Alltagskommunikation über Kunst (im weiteren Sinne) 
leistet die vorliegende Arbeit einen Beitrag, indem sie ein die bisherigen Erkenntnisse erweiterndes Spektrum an Charakteristika von Bewertungsinteraktionen sowie an sprachlichen Mitteln zur Realisierung des Bewertens aufzeigt.

Über die in bisherigen gesprächsanalytischen Studien und linguistischen Untersuchungen zum Bewerten in der Kunstkommunikation hinaus ist eine durch die Untersuchung von Bewertungsinteraktionen in den vorliegenden Gesprächen zentrale Erkenntnis der Arbeit, dass Bewertungsinteraktionen zwar mit dem Verpflichten (zum Beispiel durch Bewertungseinforderungen) und damit verbundenen Face-Bedrohungen einhergehen, aber auch mit dem Ermöglichen und Entlasten: Bewertungsinteraktionen dienen nicht nur der Aushandlung von Bewertungen, Haltungen und Positionierungen, sondern ermöglichen es auch, voneinander zu lernen, begünstigen den Abbau von Wissensasymmetrien und tragen neben der Aneignung des Rezipierten auch zur Aneignung von Praktiken der Kunstkommunikation bei. Es geht also in der innerhalb der Gesprächsgruppe stattfindenden Kunstkommunikation nicht um eine Distinktion nach innen, sondern um Vergemeinschaftung und auch um das Weitergeben von (Insider-)Wissen. Dass dabei das Pausengespräch durch theatrale Reinszenierungen, die der Bewertung oder Begründung einer Bewertung dienen können, als Bühne genutzt wird, zeigt, dass es im Rahmen geselliger Interaktion und auch im Rahmen von Bewertungsinteraktionen ebenso um das Sich-Vergnügen geht. Das Vergnügliche am Bewerten und damit auch an der sozialen Veranstaltung des Theaterbesuchs wird so von den Beteiligten selbst gemeinsam kommunikativ etabliert (vgl. Klemm 2001: 105; siehe auch Kapitel 3.1). Die Theaterpause als Bestandteil der Instituion des Theaters ermöglicht es den Beteiligten, Anschlusskommunikation über das Rezipierte zu betreiben, und kann sie auch von Zugzwängen entlasten, die mit der Kunstkommunikation verbunden sind. Schließlich können sich die Beteiligten auch selbst innerhalb der Bewertungsinteraktionen entlasten, indem sie einen Themenwechsel (auf ein anderes Bezugsobjekt) initiieren und so neue Beteiligungsmöglichkeiten schaffen.

Mit den vorangehend dargestellten Ergebnissen bildet die Arbeit zugleich einen Ausgangspunkt für weitere Untersuchungen: Um das Erhebungsdesign möglichst wenig invasiv zu gestalten, wurde auf Videoaufnahmen verzichtet und auf Audioaufnahmen zurückgegriffen (vgl. Kapitel 6.3). Entsprechend konnten bei der Analyse der Bewertungsinteraktionen lediglich verbale und paraverbale Ausprägungen berücksichtigt werden. Aufgrund der prosodischen Konturierung einiger Bewertungen beziehungsweise Bewertungseinforderungen in den analysierten Beispielen ist davon auszugehen, dass Bewertungen oder Bewertungseinforderungen nicht nur durch explizit verbale sowie paraverbale Elemente realisiert werden, sondern dass ebenso nonverbal Wertung indiziert werden kann, ohne diese verbalisieren zu müssen. Anknüpfend an die in dieser Arbeit durchgeführten Analysen 
würde sich eine multimodale Analyse des vorliegenden Gesprächstyps anbieten, um - ähnlich wie Lindström und Mondada (2009) - herausstellen zu können, wie auch körperlich (beispielsweise durch Mimik, Gestik und Blick) Bewertungen realisiert werden.

In der Forschung zur Kunstkommunikation versuchen jüngere Studien (vgl. Müller/Stegmeier 2016) nachzuzeichnen, wie Kunstkommunikation und -aneignung nicht nur in situ vor dem Kunstwerk und interaktiv mit anderen, sondern auch online stattfinden kann, indem sich die Betrachter beispielsweise durch ein Selbstportrait vor dem Kunstwerk, begleitet von einem kurzen Statement und/oder Hashtags in sozialen Netzwerken positionieren. Hier wäre ebenfalls eine sich an die in dieser Arbeit gewonnenen Erkenntnisse anschließende Untersuchung denkbar, die nach spezifischen Ausprägungen von Bewertungen und Bewertungsinteraktionen fragt, die in der online stattfindenden Kunstkommunikation im Anschluss an die Rezeption eines Theaterstücks realisiert werden.

Schließlich wäre in Anlehnung an die Rezeptionsstudie von Johanson und Glow (2015) (siehe Kapitel 3.2) eine gesprächsanalytisch vorgehende Vergleichsstudie mit Teilnehmern interessant, die nicht über die Vertrautheit mit der sozialen Veranstaltung des Theaterbesuchs und Kompetenzen zu deren Bewältigung verfügen, wie dies etwa bei den Beteiligten an den in dieser Arbeit analysierten Gesprächen der Fall ist. Eine solche Studie könnte weitere Erkenntnisse für die Frage nach der Rolle des Publikums für das Theater bereithalten. 
